\title{
INTEGRATING KINEMATIC- AND VISION-BASED INFORMATION TO BETTER UNDERSTAND DRIVING BEHAVIOUR
}

\author{
Oren Musicant ${ }^{1}$, Tomer Toledo ${ }^{2}$, Tsippy Lotan $^{3}$, Einat Grimberg ${ }^{3}$, \& Dov Ganor ${ }^{3}$ \\ ${ }^{1}$ Department of Statistics and Operations Research, Tel-Aviv University, Tel-Aviv, Israel \\ ${ }^{2}$ Civil and Environmental Engineering, Technion-Israel Institute of Technology, Haifa, Israel \\ ${ }^{3}$ Or Yarok, Hod Hasharon, Israel \\ Email: musicant.oren@gmail.com
}

\begin{abstract}
Summary: This study explored the use of two types of advanced driver assistance systems (ADAS) as tools for observing driving behavior. The first was a kinematic-based ADAS that uses speed and acceleration data to detect driving events such as hard braking, speeding and sharp turning. The second was a visionbased ADAS that uses video data to provide lane departure warnings (LDW), headway warnings (HW) and forward collision warnings (FCW). Data was collected for more than 4,500 trips and 2,200 driving hours during a period of 70 days. The sample consisted of 10 drivers that used both types of ADAS simultaneously. The information collected also included more than 17,000 records of various types of driving events. First, the events rates were estimated by the Poisson and the Poisson-lognormal models. Then, Pearson correlation and factor analysis were implemented to study the relationships among the events and to evaluate whether different types of events converged to describe the same behaviors. Significant correlations were observed between the braking and turning kinematic-based events and the FCW vision-based event, which converged under the same factor. High rates of these events may indicate that the person is driving in an urban style. The LDW, HW and speeding events converged to the second factor, which is more relevant in inter-urban areas. These findings, although based on a small-scale study, point to a potential for the use of commercial ADAS for driving behavior analysis. The integration of kinematic-based and vision-based information can provide deeper understanding of the measured behavior.
\end{abstract}

\section{INTRODUCTION}

Advanced driver assistance systems (ADAS) aim at decreasing undesirable or unsafe driving behaviors, thus reducing the probability of crash involvement. This study focuses on the analysis of information provided by two different types of commercially available ADAS. The first type is an in-vehicle collision avoidance warning system (IVCAWS) which uses vision sensors to detect risky situations (such as close proximity to a leading vehicle and unintended lane drifting) that require the driver to take preventive action in order to avoid a crash. There is evidence that IVCAWS systems can improve driver performance, but overreliance on the warnings can negatively affect behavior (Maltz \& Shinar, 2007).

Another type of driver assistance system uses kinematic data (such as acceleration and speed) to detect and report on the occurrence of various driving events such as hard braking and speeding. Rather than provide warnings to prevent an immediate risk from developing into a crash, these devices serve as monitoring and feedback tools to promote safety behavior. There is evidence 
that this driving event information can be used as a surrogate for safety, as it is significantly correlated to crash involvement (Toledo, Musicant, \& Lotan, 2008). Researchers have also found the information logged by them helpful in discovering new information about behavior. For example, the rate of undesirable driving events was found to be higher at the beginning and end of trips (Musicant, Bar-Gera and Schechtman, 2010) and for young drivers with sensationseeking tendencies (Prato, Lotan and Toledo, 2010). Parental behavior was linked to novice driver behavior, and parental involvement was linked to a reduction in the events rate (McGehee, et al., 2007, Prato, Lotan and Toledo, 2009).

The study presented in this paper was conducted as part of the PROLOGUE project conducted within the European Union's Seventh Framework Programme (EU FP7) (Lotan et. al. 2010). This study explored the potential of these two ADAS technologies as observational tools for driving behavior. Specifically, the objectives are to provide an initial understanding of the methods that may be used to analyze the rich data that these systems provide, as well as to explore the extent that the data collected by the two systems is similar or complimentary in helping to understand driving behavior.

\section{METHOD}

In this study, the GreenRoad (GR) kinematic-based system and the Mobileye (ME) vision-based IVCAWS were used to measure driving behavior. The GR system identifies various undesirable driving events such as swift lane changes, sharp turns, sudden braking, strong acceleration and high speeds by implementing pattern recognition algorithms to the raw speed and acceleration profiles. The information is transmitted to an application server, along with additional information about the trip start and end time. A complete description of this system is presented in Toledo et. al.2008.

The ME technology feeds video data from a single camera located on the front windshield inside the vehicle and inputs from the vehicle (such as vehicle speed sensors, signals and brakes) to a specialized processing unit. The processing unit uses image-processing algorithms to provide lane departure warning (LDW), headway warning (HW - based on distance to the vehicle in front) and forward collision warning (FCW - based on time-to-collision estimates). The technical specs of the hardware, along with a detailed description of the system, can be found on the Mobileye website (Mobileye, 2010). Unlike the GR system, ME does not have built-in capabilities to transmit and store data and to detect the beginning and end of a trip, which are needed to control for exposure. Therefore, the ME device was integrated with another off-theshelf car tracking device called TrackTec (TT). The TT system was modified, enhanced and calibrated to support logging of the events detected by ME.

Ten volunteers (seven male and three female) participated in a 70-day data collection period. Both the ME and GR systems were installed in each volunteer's vehicle. Participants were employed by the road safety organization Or Yarok. Therefore, all drivers were highly aware of safe driving practices. They all had at least 15 years of driving experience, and drove at least 1.5 hours a day, on both urban and inter-urban roads. 
An initial screening process discovered data quality issues. In certain cases, the TT stopped recording $\mathrm{ME}$ alerts, but reported trip start and end times. This phenomenon was visible when exploring the events rate by days from the experiment start time, where at some point the event rate becomes zero until the end of the experiment. This was likely caused by a technical problem rather than actual behavior. Therefore, for the evaluation of the events rate, data was used until the date of the last recorded ME event for each vehicle. In addition, the reporting of very short trips (less than 2 minutes) was much more common with the TT device than with the GR device. We assume this was mostly due to different methods implemented to identify trips' start and end. Thus, for the comparison between the two systems, we considered only trips with durations of 2 to 300 minutes, which represent over $85 \%$ of the trips recorded by each of the systems. In one of the vehicles, a technical problem caused an inflation of FCW events relative to the other vehicles (3010, as compared to an average of 305). This vehicle's FCW data was disregarded. Thus, the analysis refers to 9 out of 10 drivers, according to data availability.

Our variable of interest was the events rate given by the count of driving events per minute of driving. Two methods were used to estimate the logarithm of the events rate: the Poisson regression model and the multivariate Poisson-lognormal mixed effect regression model. The Poisson regression model was defined as follows:

$$
\left.\ln \left(E_{\left(\text {Events }_{i j}\right.}\right)\right)=\ln \left(\text { Duration }_{i j}\right)+\beta_{j}
$$

Where Events $_{i j}$ and Duration $_{i j}$ are the count of events and the duration in minutes of trip $i$ of driver $j . \beta_{j}$ is a driver-specific constant for the ln (events rate).

The Poisson-lognormal mixed effect regression model assumes that the driver-specific parameters $\left(\beta_{j}\right)$ are drawn from a normal distribution.

For the purpose of this study, it was interesting to analyze how the events interact, especially with regard to the linkage between the GR and ME events rates. First, we studied the correlation between all events generated by the two systems. Then we implemented factor analysis over the variables in order to evaluate how different events converged under the same factor to describe behaviors that cannot be measured directly. For the factor analysis, we used a principle component analysis (PCA) and Varimax rotation method.

It should be noted that, due to the small sample size (10 drivers), the results cannot be considered robust for generalization purposes. Instead, our results should be discussed with regard to analysis methods and towards assessing the potential of using off-the-shelf low-cost devices to identify, and better understand, driving behavior.

\section{RESULTS}

\section{Exposure}

The number of trips and time driven were recorded by the two systems. The measures obtained from the two systems did not fully match. In total, TT recorded more trips (TT: 2681 vs. GR: 
2128) and more driving time (TT: $1141.32_{\text {hrs }}$ vs. GR: $1110.67 \mathrm{hrs}$ ) than GR. This result is attributed to different protocols used to detect trip start and end times.

Figure 1 shows the relations between the measurements provided by the two systems. The 45degree red line represents a perfect match between the measurements. High correlations in the trip counts $(\mathrm{r}=0.94, \mathrm{p}<0.001)$ and the driving time $(\mathrm{r}=0.99, \mathrm{p}<0.001)$ were observed between the two systems across vehicles.
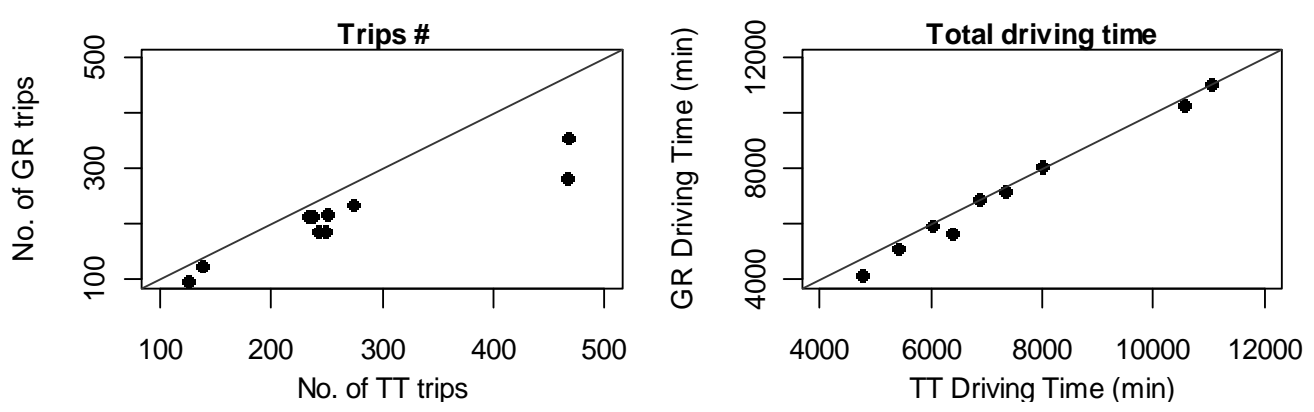

Figure 1. Relation between numbers of trips and driving times measured by the two systems

In practice, measurements provided by different systems are not expected to be exactly identical. The differences can be attributed to the different definitions of trip start and end. The overall exposure differences are considered minor, especially when looking at the driving time.

\section{Events Counts and Rates}

In total, there were 16,711 ME events, among which the most frequent were HW (9922 events), followed by LDW (4039 events) and FCW (2750). GR reported 707 events, the most frequent of which were turning (239), speeding (223) and braking (221). Lane changing (16) and accelerating (8) events were relatively rare and so were not considered informative for distinguishing among drivers in our sample.

The estimated events rates (events per minute) are presented in

Figure 2 for each driver and event type. The black points and lines represent the Poisson regression fitted values, and their corresponding confidence intervals. The red points represent the estimates obtained from the Poisson-lognormal regression model. The two models produce very similar results. Due to the large amount of data (i.e. number of events), especially for the ME events, there is high certainty (narrow confidence intervals) in the rate estimates. Therefore, the Poisson-lognormal model did not cause substantial changes to the individual rates in the direction of the average across vehicles. The GR events are less common, so the estimation of the Poisson model had less certainty (wider confidence intervals), making the difference between the two models' estimates more pronounced. This is more pronounced for the speeding events, which are very rare in our samples, and especially for drivers 5, 7 and 10 (see Figure 2). In light of these results, we decided to use the Poisson-lognormal model. 

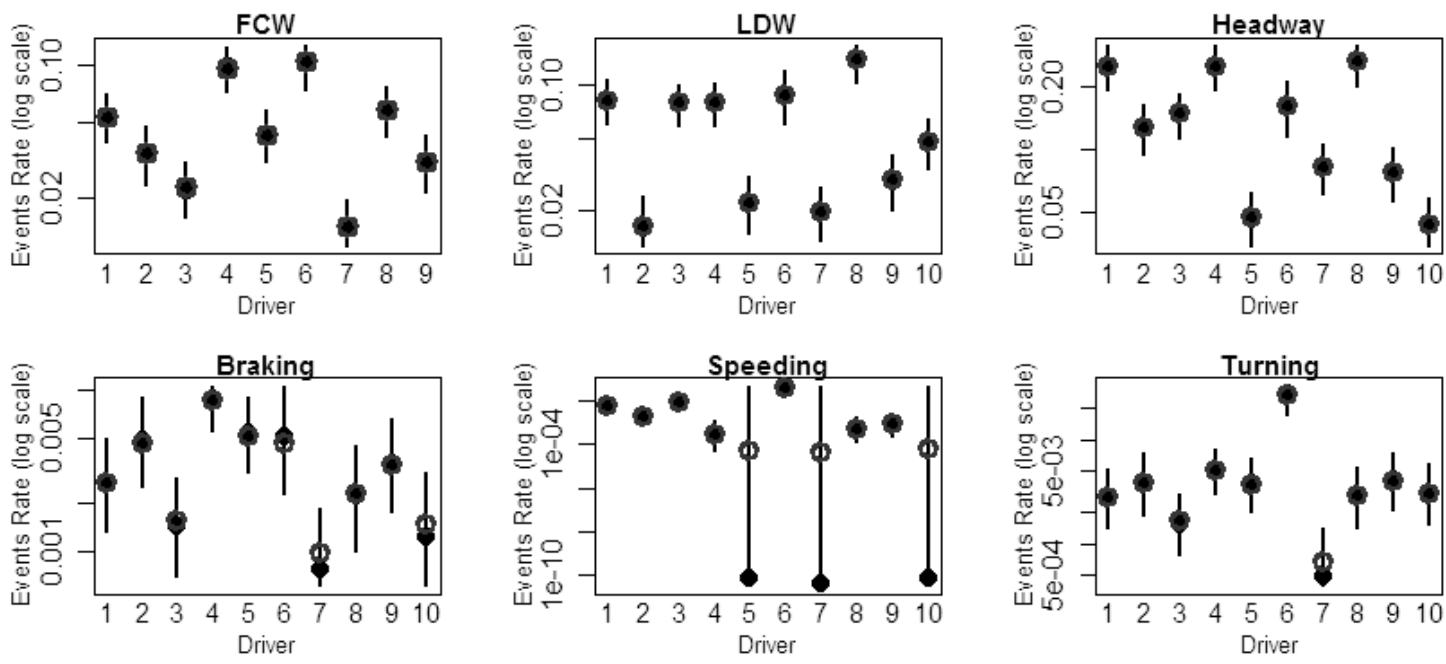

Figure 2. Expected values of events rates

The estimated events rates by type are presented in the box-plot in Figure 3. Once again, it is clear that GR events are much less frequent than ME events. The variability among drivers is also interesting, as an event that exhibits higher variability across drivers may be more useful when trying to differentiate among drivers. The events with the largest variability are speed and LDW. Variables with relatively low variability are turn handling, FCW and braking.

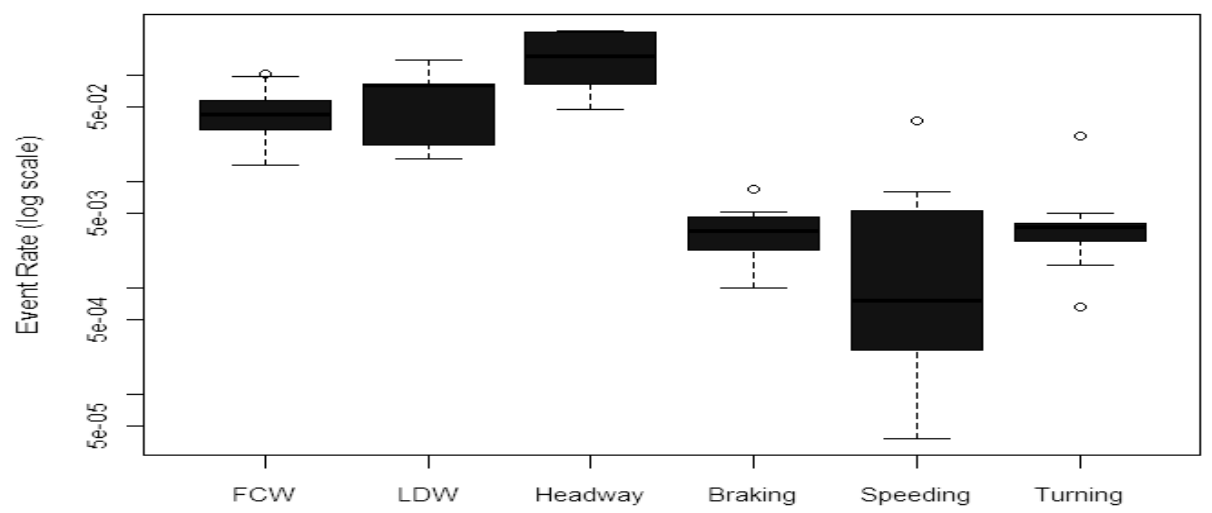

Figure 3. Estimated events rates by type

\section{Relations among various events}

The two systems used in this study measure different driving events. However, it is unclear to what extent the various events are complementary or represent different measurements or manifestations of the same behaviors. To examine that, we studied the Pearson correlations (Table 1) between the various events' estimated ln (events rates). In all cases, the correlations scores are positive. The correlations among ME events are relatively high, where the correlation between HW and LDW is statistically significant $(r=0.679, \mathrm{p}<0.05)$. For the GR events, the high correlation between braking and turn handling is also significant $(\mathrm{r}=0.696, \mathrm{p}<0.05)$. 
The correlations between ME and GR events are particularly interesting. There is a high correlation between FCW, braking and turning $(r=0.763$ and $r=0.833, p<0.05)$. The correlation between speed events and FCW is not significant but achieved positive scores similar to the correlations between speed and braking (and turning). We attribute the lack of significance to the small sample size and not to the actual differences between the devices. The correlations found between the various events raised the question of whether some latent behaviors are manifested by different types of events. To investigate this, an exploratory factor analysis (PCA+Varimax) was conducted over the six event types. A two-factor solution (Table 1) accounted for $82 \%$ of the variance. The FCW, braking and turning event types had high loadings to the first factor. High rates of braking and turning can indicate a high exposure to urban driving, where there are many intersections and traffic signs. Most FCW events occur at low speeds, which may reinforce the assumption that the first factor was related to urban driving. The second factor included LDW, HW and speed. The speeding event is more likely to occur on highways, as it is defined as speed exceeding a high threshold (120km/h in our case). LDW and HW are also more relevant in inter-urban areas. Therefore, the second factor may represent inter-urban driving.

Table 1. Correlations and factor analysis (PCA+Varimax) results

\begin{tabular}{|c|c|c|c|c|c|c|c|c|}
\hline & \multicolumn{6}{|c|}{ Correlations among event rates of the various event types } & \multicolumn{2}{|c|}{ Factor analysis } \\
\hline & FCW & LDW & HW & Braking & Speeding & Turn & Factor 1 & Factor 2 \\
\hline FCW & & 0.588 & 0.532 & $0.763 *$ & 0.380 & $0.833^{* *}$ & $\underline{0.84}$ & 0.47 \\
\hline LDW & & & $0.679 *$ & 0.025 & 0.475 & 0.310 & 0.11 & $\underline{0.92}$ \\
\hline HW & & & & 0.252 & 0.584 & 0.184 & 0.07 & $\underline{0.90}$ \\
\hline Braking & & & & & 0.205 & $0.696 *$ & $\underline{0.94}$ & -0.08 \\
\hline Speed & & & & & & 0.541 & 0.25 & $\underline{0.70}$ \\
\hline Turn & & & & & & & $\underline{0.90}$ & 0.26 \\
\hline SS & & & & & & & 2.49 & 2.45 \\
\hline $\begin{array}{l}\text { Proportion of the } \\
\text { variance }\end{array}$ & & & & & & & 0.42 & 0.41 \\
\hline
\end{tabular}

\section{DISCUSSION}

This study explored the use of commercially available vision-based and kinematic-based ADAS to detect and report driving events. The study demonstrates our ability to extract both meaningful and rich driving information from these systems. The two systems used in this study reported similar exposure variables, yet there was a large difference in the number and type of events recorded. The analysis performed suggests that the measurements are complimentary, at least to some extent.

The use of off-the-shelf products for research raises the issue of system configuration. In many cases, ADAS systems are a "black box" in which the algorithms used, definitions of driving events, and threshold values are not explicitly known or are sometimes hardwired in the equipment and cannot be changed. It is important to be able to configure the systems so that meaningful information can be collected. 
For the statistical analysis, the Poisson-lognormal estimations were preferred because they were more "conservative" (i.e., close to the average) compared to the more simple Poisson model that was, in some cases, extreme (i.e., far from the average) but based on small counts. With regard to the correlation analysis, despite the small sample size, some of the correlations were statistically significant. Therefore, the measurements in our sample are complimentary, at least to some extent. The factor analysis suggests that various events indicate underlying factors that are not specifically related to the measurements made by each system. The two factors we extracted accounted for urban and inter-urban driving. We should emphasize that the results in this small sample size cannot be generalized. However, they do suggest that there is a feasible potential for studies with larger samples to achieve deeper understanding of driver behavior by using a synthesis of kinematic- and vision-based driving events information.

Future studies can examine events that were recorded together during the same trip and in temporal proximity. This type of analysis may help focus on interesting driving situations, as well as provide insight into the evolvement of driver action in certain situations. Another potential direction is to use the most severe events recorded in the data as proxies for near-crash situations.

\section{REFERENCES}

Lotan, T., Albert, G., Ben-Bassat, T., Ganor, D., Grimberg, E., Musicant, O., Hakkert, S. and Toledo T. (2010). Israeli Field Trial. PROLOGUE Deliverable D3.2. Or Yarok, Hod Hasharon, Israel.

Maltz, M., \& Shinar, D. (2007). Imperfect in-vehicle collision avoidance warning systems can aid distracted drivers. Transportation Research Part F 10 , 345-357.

Mobileye. (2010). Retrieved November 15, 2010, from http://www.mobileye.com/manufacturerproducts/white-papers.

Musicant, O., \& Lampel, L. (2010). When Technology Tells Novice Drivers How to Drive. TRB 89th Annual Meeting.

Musicant, O., Bar-Gera, H., \& Schechtman, E. (2010). Electronic records of undesirable driving events. Transportation Research Part F 13(2), 71-79.

Prato, Carlo Giacomo, Tsippy Lotan, and Tomer Toledo. "Intra-familial transmission of driving behavior: evidence from in-vehicle data recorders." TRB Annual Meeting. 2009.

Prato, C., Toledo, T., Lotan, T \& Taubman - Ben-Ari O. (2010). Modeling the behaviour of novice young drivers during the first year after licensure. Accident Analysis and Prevention, 42 (2), 480-486.

Toledo, T., Musicant, O., \& Lotan, T. (2008). In-vehicle data recorders for monitoring and feedback on drivers' behaviour. Transportation Research Part C ,16, pp. 320-331. 